\title{
Robot-assisted brachytherapy of the bladder with long distance support using video conferencing
}

\author{
Francisco Mascarenhas, MD', Kris Maes, MD²,3, Fernando Marques, MSc', Rui Formoso, MD²,3, Telma Antunes, MD' \\ 'Radiation Oncology Department, ${ }^{2}$ Urology Department, ${ }^{3}$ Robotic and Minimally Invasive Surgery Center, Hospital da Luz, Lisboa, Portugal
}

\begin{abstract}
Purpose: The frontline treatment for localized muscle-invasive bladder carcinoma (MIBC) is radical cystectomy. However, a significant percentage of this population is elderly with either severe co-morbidities or suboptimal general health, increasing the per- and post-operative risk when undergoing a radical cystectomy. Conservative treatment options have been implemented such as robot-assisted laparoscopic brachytherapy (RALB), a minimally invasive therapeutic approach ensuring excellent results in terms of local control, survival, and low morbidity. The treatment was supported successfully long distance using videoconferencing by an expert group from the Netherlands.

Material and methods: An 81-year-old man was treated with RALB. The patient was submitted to external beam radiotherapy (EBRT) in a total dose of $40 \mathrm{~Gy}$ in 20 fractions. A partial cystectomy followed by implantation of three catheters along the scar and brachytherapy was completed. A total dose of $25 \mathrm{~Gy}$ in 10 fractions was administered with dose points calculated at $5 \mathrm{~mm}$ from the catheters.

Results: Dose planning was characterized by an homogeneity index of $62.3 \%$ and overdose index of $19.7 \%$. Other conformity indexes were analyzed. Patient was discharged at the fourth day after the procedure. The clinical response after 6 months was documented.

Conclusions: The previously reported good rates of tumor control and survival, and the excellent tolerance and low morbidity support RALB as a promising approach for selected groups of patients with localized MIBC. This case illustrates the ability to expand efficiently this technique, particularly among inexperienced medical teams.

Key words: bladder cancer, robotic brachytherapy, video-conferencing.

\section{Purpose}

Bladder cancer is a common disease on the elderly with a peak incidence at 75 years [1]. The higher age and concomitant comorbidities are usually associated with the use of tobacco and constrains the treatment options in these patients. The standard treatment of localized muscle invasive bladder cancer (MIBC) is still considered to be a radical cystectomy with pelvic lymph node dissection. The combined modality therapy (CMT) for bladder preservation with neoadjuvant chemotherapy, transurethral resection of bladder tumor (TURBT), or partial cystectomy and external beam radiotherapy (EBRT), results in low rate of late relapses and similar survival outcomes in long-term survivors [2]. Another effective, safe, and less toxic treatment option in the context of bladder sparing, which has been a treatment option for several decades in selected patients, is the trimodality therapy (TMT) consisting of minimal surgery, including TURBT or partial cystectomy, EBRT and brachytherapy $[3,4,5,6,7]$. In 2009, bladder surgery has become advanced with the introduction of robot-assisted laparoscopic procedures combined with high-dose-rate brachytherapy $[8,9]$.
The authors describe the first patient with MIBC being treated in Portugal with bladder-sparing treatment integrating robot-assisted laparoscopic brachytherapy (RALB) with the main aim to demonstrate the ability to efficiently expand this technique, notably among inexperienced medical teams, using videoconferencing support of an expert team at long distance.

\section{Case report}

\section{Pre-operative evaluation}

An 81-year-old male, with non-insulin dependent diabetes and hypertension, was admitted in another institution with symptoms, which had evolved over 4 months characterized by mild pelvic pain, frequency and nocturia at 2 to 3 hour intervals, urgency, dysuria, and moderate hematuria. Patient was assisted in other institution and had realized only an ultrasonography and a computed tomography (CT) scan, which confirmed a tumor with maximum $5 \mathrm{~cm}$ diameter located in the bladder dome with no presence of positive pelvic lymph nodes. A magnetic resonance imaging (MRI) 
or positron emission tomography/computed tomography (PET-CT) had not been requested. The patient underwent TURBT in February 2016, and the pathology proved a high grade invasive solitary urothelial carcinoma of the bladder with invasion of the muscular layer.

Chemotherapy followed by radical cystectomy was proposed, and after three cycles of carboplatin and gemcitabine, the therapy was discontinued due to severe toxicity consisting of peripheral neuropathy, anorexia, and marked weakness. The initially proposed radical cystectomy was refused by the patient.

The patient chose to have a second opinion about alternative treatment options and resorted to another center.Because of toxicity of the neoadjuvant chemotherapy experienced by the patient, he rejected a chemoradiation option. ${ }^{18}$ F-FDG PET-CT scan confirmed a metabolically active solitary tumor located in the bladder dome without other regional or distant lesions. The post-chemotherapy coronal and axial T1- and T2-weighted MRI (3 Tesla) images, and T1 VIBE FS post-gadolinium sagittal images showed a remaining solid lesion of the bladder dome mea- suring $3.8 \mathrm{~cm} \times 2.2 \mathrm{~cm}$, with some alteration of the signal of the deep bladder wall, suggesting a T2bN0M0 bladder tumor (Figure 1).

Considering advanced age of the patient, co-morbidities, inherent life expectancy, refusal to undergo radical cystectomy, and the fact that the tumor fulfilled the selection criteria of the GEC-ESTRO recommendations [1,3] (Table 1), the option of a conservative treatment with minimal resection, EBRT, and brachytherapy as a boost in the context of bladder preservation was proposed. The patient and his relatives, very concerned about toxicity, were informed about this strategy including robotic surgery by a skilled team, as the most safe and accurate approach to increase the radiation dose to the residual tumor and the most conservative surgical procedure as an alternative to radical cystectomy. Informed consent was duly clarified and signed. In July 2016, the patient initially underwent complete transurethral resection of the residual bladder tumor. Pathology confirmed a persistent high-grade solitary tumor involving the muscle layer. Random biopsies showed no further disease in other bladder sites.
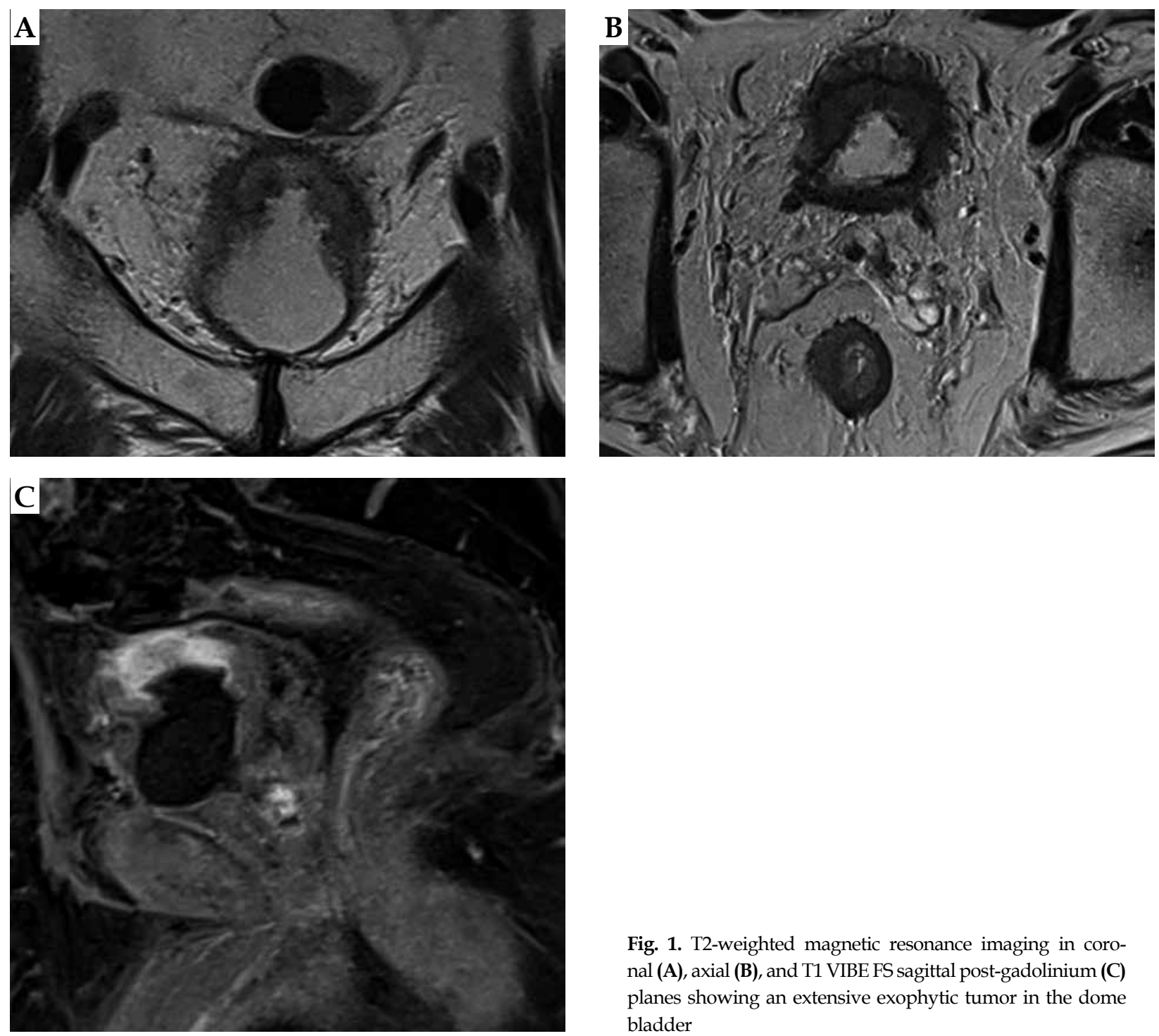

Fig. 1. T2-weighted magnetic resonance imaging in coronal (A), axial (B), and T1 VIBE FS sagittal post-gadolinium (C) planes showing an extensive exophytic tumor in the dome bladder 
Two weeks post-TURB, EBRT of 40 Gy in 20 fractions started using image-guided volumetric modulated arc therapy, with the target volume defined by the whole bladder and regional iliac lymph nodes up to the aortic bifurcation. Ten days after EBRT, a new cystoscopy and MRI revealed $9 \mathrm{~mm}$ thick tumor and absence of other suspicious locoregional lesions or lymph nodes.

\section{Brachytherapy treatment}

The surgical procedure was carried out three weeks after EBRT by the patient's wishes and contrary to the followed therapeutic protocol comprising 10 to 15 days of interval between both therapies. All treatment phases including robotic surgery, catheter implantation, and planning dosimetry were long distance supported using videoconferencing by the expert medical team of Rijnstate Hospital in Arnhem, the Netherlands, consisting of a urologist, a radiation oncologist, and a physicist. This team was able to initially assess the entire clinical process with the consent of the patient and his family and participated in the therapeutic decision prior to treatment. They had access to EBRT planning and dosimetry, the response with endoscopic evaluation and MRI images, and especially to the entire surgical procedure in real time through three monitors in the operative theater, allowing the evaluation of laparoscopic images, cystoscopy and contacting whenever necessary urologists and radiation oncologists involved.
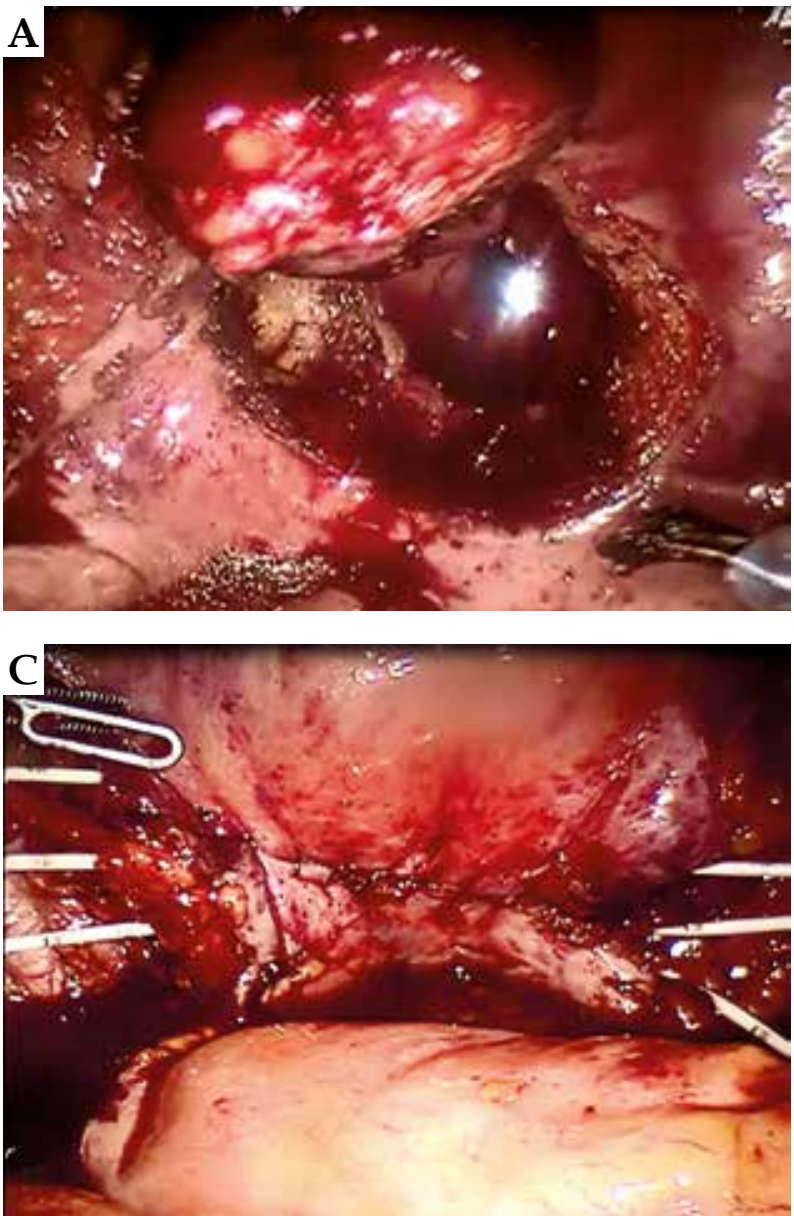

Table 1. Patient selection criteria according GEC-ESTRO recommendations $[1,3]$

1. Patients with operability criteria, tolerating a general anesthesia

2. Solitary tumor with a maximum diameter of $5 \mathrm{~cm}$

3. No concurrent carcinoma in situ elsewhere in the bladder

4. A tumor classified CT2-T3 following the UICC TNM 7 ed. classification [10]

5. Tumor not located in the bladder neck and close to prostatic urethra in male patients

6. No distant metastases

GEC-ESTRO - Groupe Européen de Curiethérapie European Society for Radiotherapy and Oncology, UICC - Union for International Cancer Control

In the Trendelenburg position, the laparoscopic camera and three robotic trocars were inserted into the abdominal cavity and subsequently connected to the Da Vinci robot. The cystoscope was also inserted to define the tumor mapping and to monitor the resection of the tumor within the bladder. The mapping of the tumor area was ensured with the aid of the light of the cystoscope being visible in the abdominal cavity via laparoscopy. Initially, lymph node dissection was performed followed by partial cystectomy with resection of the residual tumor and margins, which was

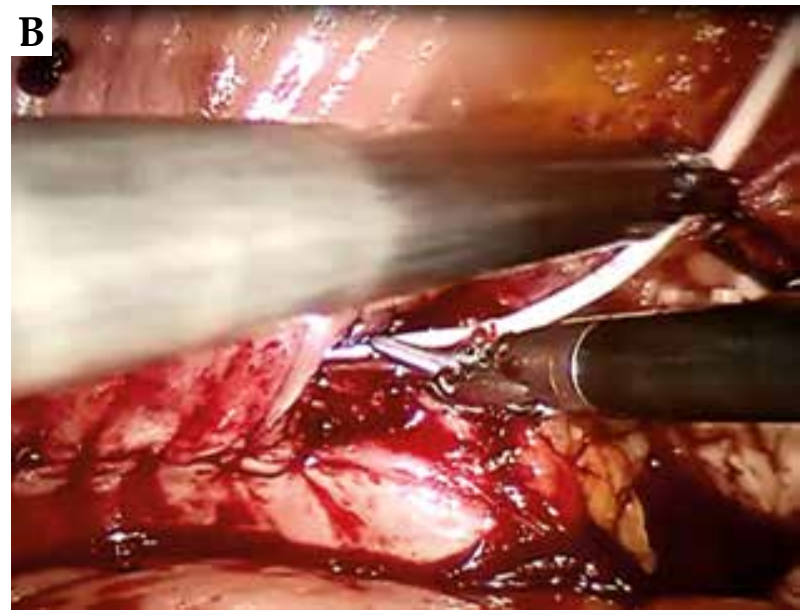

Fig. 2. A) Laparoscopic resection of bladder tumor with Da Vinci robot under cystoscopic control and after mapping lesion. B) The needle of each Luneray catheter is manipulated by the robot and implanted in the middle of the muscular layer, using cystoscopic monitoring during implantation. C) All catheters are placed with a parallel positioning and a separation of 7 to $10 \mathrm{~mm}$ longitudinally encompassing the cystectomy scar with $10 \mathrm{~mm}$ margins 
hampered by the presence of a nearby bladder diverticulum (Figure 2A). After adequate suturing, three $50 \mathrm{~cm}$ Luneray catheters with needles of $67 \mathrm{~mm}$ in length and a radius of $75 \mathrm{~mm}$ required to involve whole length of the scar, specifically developed for this procedure (Nucletron, an Elekta company, Elekta AB, Stockholm, Sweden) were implanted. The radiation oncologist inserted the needle of each catheter through the skin of the abdomen, in a precise location according to the orientation of the bladder implant given by the urologist. The urologist positioned the catheters within the bladder wall, using cystoscope monitoring during implantation, to ensure that they remain intramural and do not enter intravesically, and also to allow the endoscopic evaluation of the implant control with regard to the extent and location of the scar (Figures 2B and C).

Each needle was then pulled out through the skin at the other side of the abdomen, and then removed from the catheter. All three catheters were implanted this way, with a parallel positioning, separated by 7 to $10 \mathrm{~mm}$, two of them placed parallel, at either side of the cystectomy scar with margins of about $5 \mathrm{~mm}$. The third was placed in between the others to ensure that the prescription isodose could adequately cover all target because of a slight divergence of the lateral catheters due to the presence of the diverticulum. Two titanium clips were placed laparoscopically with the robotic trocars at both ends of the catheters in the external wall of the bladder, to define the clinical target volume (CTV), and facilitate the visualization of the tumor area to be treated on the CT images and in the dose planning system. On both sides of the abdomen, fixating buttons were attached to the catheters and then the patient was transferred to the recovery room.

On the day of the surgery, a planning CT was made with $1 \mathrm{~mm}$ slice thickness. Fiducial markers were inserted inside the catheters enabling their reconstruction. A dose planning with source stop positions or dwell time active positions between the surgical clips were then generated using a brachytherapy treatment planning system (Oncentra Brachy, Nucletron, an Elekta company, Elekta $\mathrm{AB}$, Stockholm, Sweden). Dose points at $5 \mathrm{~mm}$ from the catheters were used and dwell time optimization was performed. In order to reduce excessive high dose regions around the catheters the dwell times were manually improved. The dose distribution was evaluated and the best plan approved, with an homogeneity index (HI) of $62.3 \%$ and an overdose index (OI) of $19.7 \%$, confirming the quality of the dose plan according the values followed by Arnhem's team ( $\mathrm{HI} \geq 50 \%$ and $\mathrm{OI} \leq 28 \%$ ) (Fig. 3) [8]. $\mathrm{HI}$ represents the size of the part of the target volume that receives $100 \%$ to $150 \%$ of the prescribed dose and is defined by $\mathrm{HI}=\left(\mathrm{V}_{100}-\mathrm{V}_{150}\right) / \mathrm{V}_{100} \times 100 \%[11,12]$. The OI represents the size of the part of the target volume that receives more than $200 \%$ of the prescribed dose and is defined by OI $=\mathrm{V}_{200} / \mathrm{V}_{100} \times 100 \%$ [12]. The first fraction was performed with this dosimetric plan and evaluated by both physicists still in video conferencing. Subsequently, a new dosimetric study with manual optimization was done considering the CTV defined as the bladder scar after partial cystectomy between clips, with $5 \mathrm{~mm}$ of margins in all directions. The CTV was encompassed by the $100 \%$ reference isodose in the next nine fractions performed in the next three days. Also, the non-involved bladder outside the CTV and the organs at risk such as small bowel loops in the vicinity of the bladder, the sigmoid, and rectum were contoured. The registered dose-volume parameters and conformity indexes are presented in Table 2. An acceptable conformity index (CI) of 0.93 was found together with a healthy tissues conformity
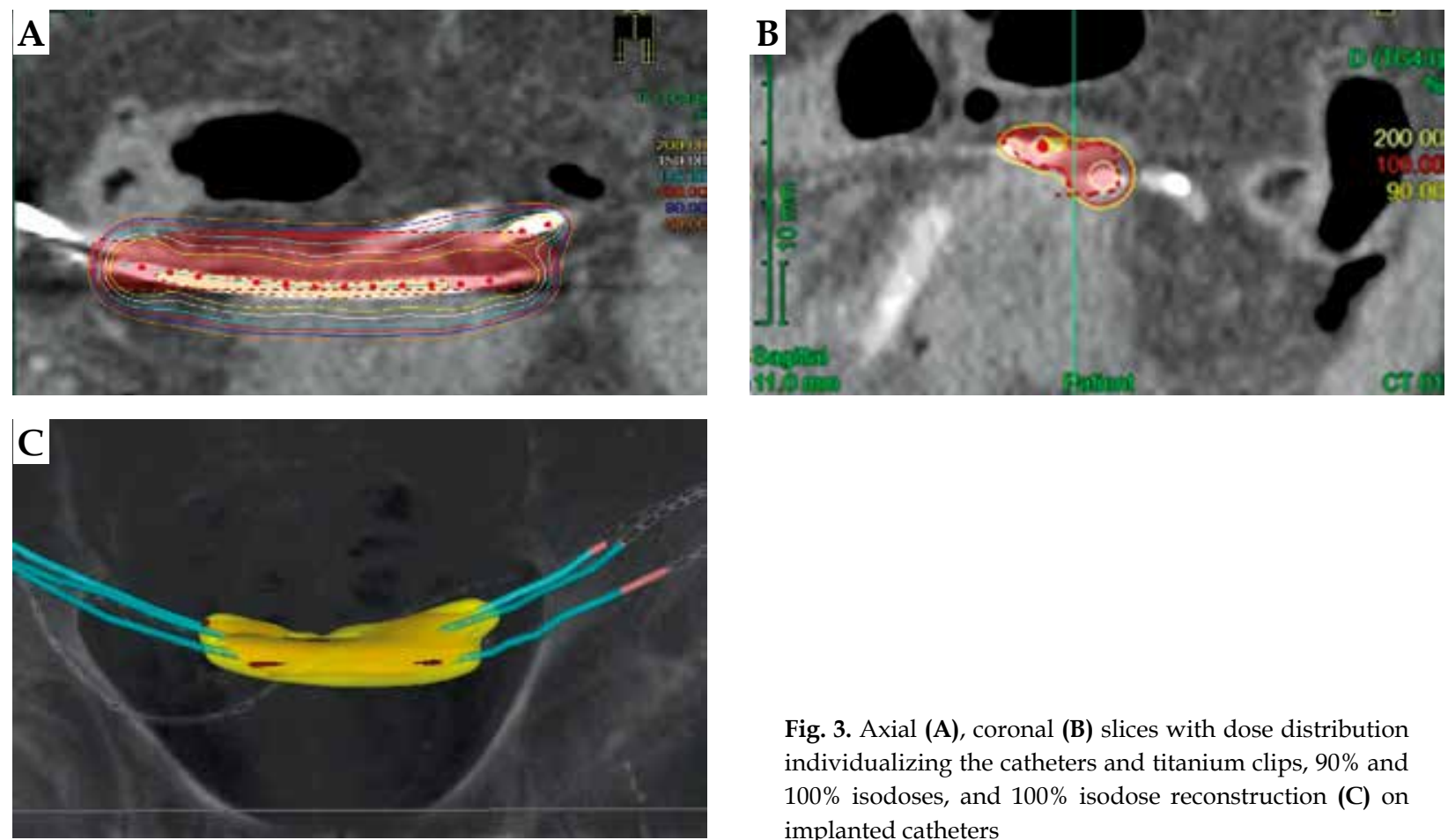

Fig. 3. Axial (A), coronal (B) slices with dose distribution individualizing the catheters and titanium clips, $90 \%$ and $100 \%$ isodoses, and $100 \%$ isodose reconstruction (C) on implanted catheters 
index (HTCI) of 0.57 [13]. This was considered a minor deviation caused by the bigger volume of the reference isodose than the target volume due the fact that the dose was prescribed to points at $5 \mathrm{~mm}$ from the source positions. The OARs received a dose far below the dose constraints.

The continuous monitoring of the whole procedure by the expert team provided the intervening team an extreme confidence, allowing a profitable discussion from the decision of partial cystectomy, the resection of larger lymph nodes and the catheters implantation. Both teams also participated actively in the brachytherapy planimetry such as dose coverage, dose homogeneity, reduction of high dose volumes and dose minimization in OAR.

\section{Postoperative course}

Brachytherapy was delivered to a total dose of 25 Gy in 10 fractions with an Iridium-192 ( $\left.{ }^{192} \mathrm{Ir}\right)$ source using an HDR afterloader (micro-SelectronHDR, Nucletron, an Elekta company, Elekta AB, Stockholm, Sweden). The first fraction was given on the day of surgery and the remaining 9 fractions on the following three days, 3 per day with a minimum interval of 4 hours. After the treatment, the catheters were removed and the patient was discharged. No intra-operative or post-operative complications were recorded, and blood loss was less than $50 \mathrm{~cm}^{3}$. Two weeks after surgery, the Foley catheter was removed. Pathology confirmed an invasive urothelial carcinoma, sized $27 \times 16 \mathrm{~mm}^{2}$, with invasion of the muscular layer and without lymphovascular or perineural invasion. The surgical margins were free above $0.8 \mathrm{~mm}$ and seven resected bilateral iliac lymph nodes were negative. The disease was staged as T2aN0R0. At three and six months follow-up, the patient has significantly improved in terms of urgency and frequency, which now occurred at 5 to 6 hour intervals. The cystoscopy revealed no evidence of other suspicious lesions and a normal mucosal surface of the remaining bladder (Figures 4A and B). T2-weighted MRI showed a mild bladder wall thickening, a post-surgical lymphocele and absence of suspicious lymph nodes (Figures $4 \mathrm{C}$ and D). Further follow-up will be performed quarterly with cystoscopy, and alternating CT and MRI imaging.

\section{Discussion}

Radical cystectomy of localized MIBC recognize an overall survival at 5 and 10 years of $60 \%$ and $45 \%$, respectively [14]. Perioperative mortality is reported in the range of $1.2 \%$ to $3.2 \%$ at 30 days, and $2.3 \%$ to $8.0 \%$ at 90 days. Numerous long-term side effects are not negligible, such as urinary dysfunction, urinary incontinence, urinary infections, and especially the loss of normal bladder function, often poorly tolerated by these patients $[15,16,17,18,19]$.

Quality of life is compromised, often significantly, even with the most modern techniques of radical cystectomy, urinary diversion, and orthotopic neo-bladder construction $[20,21]$. The CMT comprising chemoradiotherapy gives similar outcomes as RC, but avoiding associated morbidity and mortality, and thereby improving the patients' quality of life $[2,21,22,23]$.
Table 2. Brachytherapy dose-volume parameters, quality, and conformity indexes [11]

\begin{tabular}{|c|c|}
\hline Dose parameters & Values \\
\hline$V_{100}$ & $8.62 \mathrm{~cm}^{3}$ \\
\hline $\mathrm{V}_{150}$ & $4.44 \mathrm{~cm}^{3}$ \\
\hline$V_{200}$ & $2.32 \mathrm{~cm}^{3}$ \\
\hline$V_{100}$ non-involved bladder (outside of CTV) & $0.01 \mathrm{~cm}^{3}$ \\
\hline $\mathrm{D}_{2 c c}$ non-involved bladder (outside of CTV) & 142.18 cGy \\
\hline $\mathrm{D}_{0.1 \mathrm{cc}}$ non-involved bladder (outside of CTV) & 222.51 cGy \\
\hline Rectum $D_{2 \mathrm{~cm}^{3}}$ & 13.77 cGy \\
\hline Rectum $\mathrm{D}_{0.1 \mathrm{~cm}^{3}}$ & 24.35 cGy \\
\hline Sigmoid $\mathrm{D}_{2 \mathrm{~cm}^{3}}$ & 98.86 cGy \\
\hline Sigmoid $\mathrm{D}_{0.1 \mathrm{~cm}^{3}}$ & $136.21 \mathrm{cGy}$ \\
\hline Small bowell $\mathrm{D}_{2 \mathrm{~cm}^{3}}$ & 105.09 cGy \\
\hline Small bowell $D_{0.1 \mathrm{~cm}^{3}}$ & $147.77 \mathrm{cGy}$ \\
\hline Homogeneity Index (HI) ( $\geq 50 \%)$ & $62.3 \%$ \\
\hline Overdose Index (OI) ( $\leq 28 \%)$ & $19.7 \%$ \\
\hline Conformity Index (CI) & 0.92 \\
\hline Healthy Tissues Conformity Index (HTCI) & 0.57 \\
\hline Conformation Number (CN) & 0.52 \\
\hline Conformal Index (COIN) & 0.52 \\
\hline
\end{tabular}

$V_{100}, V_{150}, V_{200}$-volume of the anatomic volume receiving $100 \%, 150 \%, 200 \%$

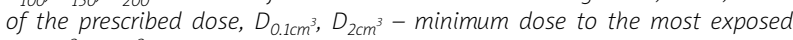
$0.1 \mathrm{~cm}^{3}, 2 \mathrm{~cm}^{3}$

Bladder cancer is more prevalent in the elderly population with severe medical comorbidities and impaired renal or hepatic function. Most of these patients are in poor general condition to undergo radical cystectomy or even to tolerate concurrent chemoradiotherapy. Chemoradiotherapy, usually including cisplatin or carboplatin concurrent with high dose EBRT, even with modern techniques such as IMRT, is less frequently but still associated with considerable risk of treatment-related toxicity and worsening of overall quality of life, particularly in patients with age-related declines in performance status and medical comorbidities who are less likely to complete CMT [24,25].

Brachytherapy centers in the Netherlands advocate for TMT, integrating a moderate dose of pelvic EBRT with a brachytherapy boost using a minimally invasive procedure in the context of organ preserving treatment for selected MIBC patients. They have demonstrated a local control and survival similar to CMT with approximately $90 \%$ of the patients cured and retaining an effectively functioning bladder $[6,8,9,23,26,27,28,29]$. Thus, the preservation of a functioning bladder remains one of the most important advantages for selected patients of all age groups.

A total dose of 40 Gy in 20 fractions of EBRT is historically administered in the Netherlands, a country with 

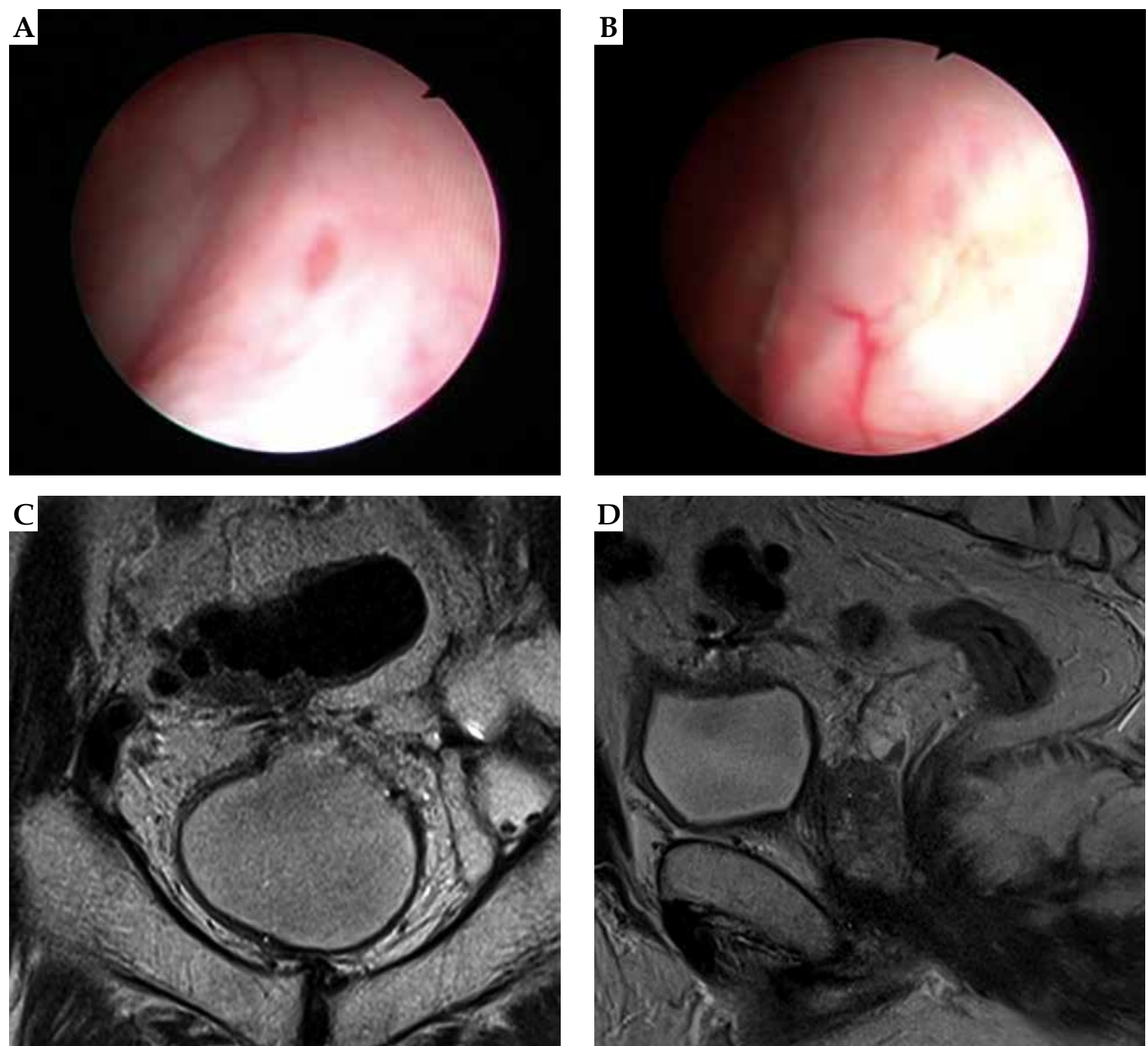

Fig. 4. Cystoscopy 6 months after treatment, showing unsuspected scar of the bed tumor and normal mucosa surface of remaining bladder (A, B). T2 weighted MRI in coronal (C) and sagital (D) planes evidencing a mild dome bladder wall thickness and a post-dissection lymphocele

a long tradition of radiation treatment for bladder cancer being considered sufficient to sterilize involved pelvic lymph nodes [30,31].

Usually if EBRT is directed to the bladder and pelvic lymph nodes, a dissection is not performed but controversy about treatment approach to pelvic lymph nodes remains [32]. In this case, there was not a significant tumor response to the EBRT and the larger pelvic lymph nodes were removed considering the potential risk of occult pelvic lymph node involvement across all clinical stages. A more accurate pre-chemotherapy staging could have allowed a more informed decision and avoided the resection of these lymph nodes.

In addition, the poor tumor response and a thick residual tumor revealed in MRI after EBRT led to the decision of partial cystectomy instead of TURB according the GEC-ESTRO recommendations [1,3].
Aluwini et al. reported long-term results of a bladder preservation strategy for MIBC using limited surgery, EBRT, and interstitial brachytherapy in 192 patients [7]. The local recurrence free survival rate was $80 \%$ and $73 \%$, and salvage cystectomy-free survival at 5 and 10 years was $93 \%$ and $85 \%$. Radiation Therapy Oncology Group grade $\geq 3$ late bladder and rectum toxicity were recorded in 11 patients $(5.7 \%)$ and 2 patients $(1 \%)$, respectively. Koning et al. published the largest cohort of 1,040 patients on brachytherapy for bladder cancer with local recurrence-free probability, metastasis-free probability, disease-free probability, and overall survival of $75 \%, 74 \%$, $61 \%$, and $63 \%$, respectively [27]. Van der Steen-Banasik et al. performed RALB with Luneray catheters in 57 patients with MIBC, 17 also underwent partial cystectomy [9]. With an average follow-up of 2 years (range, $0.5-5.8$ years), 4 patients developed bladder recurrence within the irradi- 
ated volume $(7 \%)$ and 6 elsewhere in the bladder $(10.5 \%)$. The cumulative incidence competitive risk disease free survival (DFS), disease specific survival (DSS), and local control (CI) (95\% confidence interval) at 2 years were respectively $71 \%, 87 \%$, and $82 \%$, and the overall survival was $59 \%$.

The advantages of using a brachytherapy boost over EBRT alone are the accurate delivery of radiation at the precise site with a steep dose gradient and lower toxicity due to maximal sparing of the neighboring normal organs such as the small bowel, sigmoid, rectum, uterus, vagina, femoral heads, bone marrow of the pelvis, and neural structures of the sacrum, but also the remaining normal bladder tissue. For the implementation of RALB, the introduction of dedicated, highly maneuverable catheters with great flexibility and resistance to torsion was essential. These were specifically developed by the radiation oncology and urology team from Radiotherapiegroep and Rijnstate Hospital in Arnhem, the Netherlands, in collaboration with Nucletron, now part of Elekta (LunerayTM, Elekta AB, Stockholm, Sweden), pioneering the final design in early 2014 [33]. The laparoscopic and robot-assisted brachytherapy is an emerging technique with limited experience, but with potential advantages such as minimal blood loss, reduction of hospitalization time, and the preservation of the normal bladder function, as occurred with the patient described.

The clinical team previously received appropriate training in Arnhem, and subsequently implemented the first RALB treatment with their long distance support using video conferencing. The Hospital da Luz is a paperless medical center and a model medical education at distance, implementing several clinical programs, robotic surgery technical courses, and every two years an international cancer congress with the most modern therapeutic modalities, presented in real time using video conferencing for the whole World. This model helped to receive the skills of the most experienced Dutch team and allowed the efficient implementation of this therapy for the first time in our country.

\section{Conclusions}

The presented case demonstrates the feasibility to implement robotic-assisted brachytherapy of the bladder with long distance support using video conferencing and the ability to expand this technique efficiently, particularly among inexperienced clinical teams. The authors emphasize the importance of audiovisual telementoring considering that it may play a fundamental role in future training concepts and improved impact on medical education. RALB combined with minimally invasive surgery is an attractive bladder-sparing treatment for selected patients with MIBC with fast recovery, shortening of the hospitalization, and low morbidity. The recognized similar outcomes in terms of local control and survival support this modality as a good alternative to radical cystectomy in selected population. A superior long-term quality of life in these patients is expected, but an evidence must be prospectively validated.

\section{Acknowledgment}

The authors wish to thank Elzbieta van der SteenBanasik, Geert Smits, and Bernard Oosterveld from the Rijnstate Hospital, Arnhem (the Netherlands) for their exceptional collaboration and remote treatment procedure involvement.

\section{Disclosure}

Authors report no conflict of interest.

\section{References}

1. The GEC-ESTRO handbook of brachytherapy. Van Limbergen E, Pötters R, Hoskin P, Baltas D [eds.]. Version 1 - 21/04/2015. http:/ / user-swndwmf.cld.bz/Urinary-Bladder-CancerGEC-ESTRO-Handbook-of-Brachytherapy.

2. Mak RH, Hunt D, Shipley WU et al. Long-term outcomes in patients with muscle-invasive bladder cancer after selective bladder-preserving combined-modality therapy: A pooled analysis of Radiation Therapy Oncology Group protocols 8802, 8903, 9506, 9706, 9906, and 0233. J Clin Oncol 2014; 32: 3801-3809.

3. Pieters BR, van der Steen-Banasik E, Smits GA et al. GECESTRO/ACROP recommendations for performing bladder-sparing treatment with brachytherapy for muscle-invasive bladder carcinoma. Radiother Oncol 2017; 122: 340-346.

4. Pernot M, Hubert J, Guillemin F et al. Combined surgery and brachytherapy in the treatment of some cancers of the bladder (partial cystectomy and interstitial iridium-192). Radiother Oncol 1996; 38: 115-120.

5. Wijnmaalen A, Helle PA, Koper PC et al. Muscle invasive bladder cancer treated by transurethral resection, followed by external beam radiation and interstitial iridium-192. Int J Radiat Oncol Biol Phys 1997; 39: 1043-1052.

6. Nieuwenhuijzen JA, Pos F, Moonen LMF et al. Survival after bladder-preservation with brachytherapy versus radical cystectomy. A single institution experience. Eur Urol 2005; 48: 239-245.

7. Aluwini S, van Rooij PH, Kirkels WJ et al. Bladder function preservation with brachytherapy, external beam radiation therapy, and limited surgery in bladder cancer patients: Longterm results. Int J Radiat Oncol Biol Phys 2014; 88: 611-617.

8. Nap-van Klinken A, Bus S, Janssen T et al. Interstitial brachytherapy for bladder cancer with the aid of laparoscopy. J Contemp Brachytherapy 2014; 6: 313-317.

9. Van der Steen-Banasik E, Smits G, Oosterveld B et al. The Curie-Da Vinci Connection: 5-Years' experience with Laparoscopic (Robot-Assisted) Implantation for High-Dose-Rate Brachytherapy of solitary T2 bladder tumors. Int J Radiat Oncol Biol Phys 2016; 95: 1439-1442.

10. UICC. TNM classification of malignant tumours. $7^{\text {th }}$ ed. WileyBlackwell, New York 2009.

11. Mould RF, Battermann JJ, Martinez AA. Brachytherapy from radium to optimization. Nucletron International B.V. 1994; 303-305.

12. Saw CB, Suntharalingam N. Quantitative assessment of interstitial implants. Int J Radiat Oncol Biol Phys 1991; 20: 135-139.

13. Feuvret L, Noël G, Mazeron J, Bey P. Conformity Index: A review. Int J Radiat Oncol Biol Phys 2006; 64: 333-342.

14. Finks JF, Osborne NH, Birkmeyer JD. Trends in hospital volume and operative mortality for high-risk surgery. N Engl J Med 2011; 364: 2128-2137.

15. Ravi P, Bianchi M, Hansen J et al. Benefit in regionalisation of care for patients treated with radical cystectomy: a nationwide inpatient sample analysis. BJU Int 2014; 113: 733-740. 
16. Corcoran AT, Handorf E, Canter D et al. Variation in performance of candidate surgical quality measures for muscle-invasive bladder cancer by hospital type. BJU Int 2015; 115: 230-237.

17. Henningsohn L, Steven K, Kallestrup EB et al. Distressful symptoms and well-being after radical cystectomy and orthotopic bladder substitution compared with a matched control population. J Urol 2002; 168: 168-174.

18. Hautmann RE, de Petriconi RC, Volkmer BG. Lessons learned from 1,000 neobladders: the 90-day complication rate. J Urol 2010; 184: 990-994, quiz 1235.

19. Shabsigh A, Korets R, Vora KC et al. Defining early morbidity of radical cystectomy for patients with bladder cancer using a standardized reporting methodology. Eur Urol 2009; 55: 164-176.

20. Yang LS, Shan BL, Shan LL et al. A systematic review and meta-analysis of quality of life outcomes after radical cystectomy for bladder cancer. Surg Oncol 2016; 25: 281-297.

21. Mak K, Smith A, Eidelman A et al. Quality of life in longterm survivors of muscle-invasive bladder cancer. Int J Radiat Oncol Biol Phys 2016; 96: 1028-1036.

22. Huddart R, Birtle A, Lewis $R$ et al. Results of the SPARE Feasibility Study - Selective Bladder Preservation Against Radical Excision in Muscle Invasive T2/T3 Transitional Cell Carcinoma of the Bladder (CRUK 07/011). Int J Radiat Oncol Biol Phys 2012; 84: S119-S20.

23. Blank LE, Koedooder K, van Os $\mathrm{R}$ et al. Results of bladder-conserving treatment, consisting of brachytherapy combined with limited surgery and external beam radiotherapy, for patients with solitary T1-T3 bladder tumours less than $5 \mathrm{~cm}$ in diameter. Int J Radiat Oncol Biol Phys 2007; 69: 454-458.

24. Guancial E, Roussel B, Bergsma D et al. Bladder cancer in the elderly patient: challenges and solutions. Clin Interv Aging 2015; 10: 939-949.

25. Gogna N, Matthews J, Turner S et al. Efficacy and tolerability of concurrent weekly low dose cisplatin during radiation treatment of localised muscle invasive bladder transitional cell carcinoma: a report of two sequential Phase II studies from the TransTasman Radiation Oncology Group. Radiother Oncol 2006; 81: 9-17.

26. Bos MK, Marmolejo RO, Rasch C et al. Bladder preservation with brachytherapy compared to cystectomy for T1-T3 muscle-invasive bladder cancer: a systematic review. J Contemp Brachytherapy 2014; 6, 2: 191-199.

27. Koning CC, Blank LE, Koedooder C et al. Brachytherapy after external beam radiotherapy and limited surgery preserves bladders for patients with solitary pT1-pT3 bladder tumors. Ann Oncol 2012; 23: 2948-2953.

28. Van der Steen-Banasik EM, Visser AG, Reinders JG et al. Saving bladders with brachytherapy: implantation technique and results. Int J Radiat Oncol Biol Phys 2002; 53: 622-629.

29. Dutch national workgroup for bladder cancer guidelines (2011). Available from: http://www.oncoline.nl/urotheelcarcinoom-van-de-blaas. Accessed: July 2014.

30. Van der Werf-Messing B, van Putten W. Carcinoma of the urinary bladder category T2,3NxM0 treated by 40 Gy external irradiation followed by Caesium 137 implant at reduced dose (50\%). Int J Radiat Oncol Biol Phys 1989; 16: 369-371.

31. Van der Werf-Messing B, Menon RS, Hop WC. Carcinoma of the urinary bladder category T2, T3 (NxM0) treated by the combination of radium implant and external irradiation: Second report. Int J Radiat Oncol Biol Phys 1983; 9: 481-485.

32. Goldsmith B, Baumann B, He J et al. Occult pelvic lymph node involvement in bladder cancer: implications for definitive radiation. Int J Radiat Oncol Biol Phys 2014; 88: 603-610.

33. https:/ / www.elekta.com/press/3476fb7f-d2ec-424a-95a315595588b894/innovative-luneray-catheters-and-organ-preserving-approach-result-in-radically-less-invasive-bladder-cancer-treatment.html. 